\title{
CYSTIC PERIVENTRICULAR LEUKOMALACIA: A DECLINING SOURCE OF SEVERE NEURODEVELOPMENTAL IMPAIRMENT IN PRETERM INFANTS?
}

\author{
B. Resch, E. Resch, U. Maurer, W. Müller \\ Medical University of Graz, Graz, Austria
}

Background: Cystic periventricular leukomalacia (cPVL) is one of the dreaded complications of preterm birth leading to cerebral palsy in the majority of afflicted children. We aimed to analyse local incidence rates over a 20 years time period.

Methods: Retrospective cohort analysis at a tertiary care university NICU comparing two decades.

Results: During the study period 145 of 6200 preterm infants $(2.3 \%) \leq 35$ weeks gestational age (median gestational age 30.8 weeks, birth weight 1441 grams) were diagnosed as having cPVL. There was a mean incidence rate of $3.1 \%$ (98 of 3187 infants) - varying between 1.0 and $5.8 \%$ per year - during observational period 1 (1988 to 1998) compared to $1.5 \%$ (59 of 3103 infants, $p<0.0014$ ) during period 2 (1999 to 2008) varying between 0.9 and $2.9 \%$. During period 2 we still recognized a trend towards decreasing rates of cPVL (36 out of 1616 infants between 1999 and 2003 - 2.2 \% - compared to 23 out of 1487 infants between 2004 and $2008-1.5 \%, \mathrm{p}=0.083$ ). Some perinatal data changed significantly during these two observational periods (comparing period 1 to 2 ) as shown in the table. Neurodevelopmental follow-up revealed unchanged rates of cerebral palsy ( 78.6 to $81.4 \%, \mathrm{p}=0.411)$, but significantly reduced rates of mental retardation $(61.2$ to $30.5 \%, \mathrm{p}<0.0001)$.

Conclusion: During these two observational periods we observed a significant reduction in rates of cPVL. Rates of cerebral palsy remained unchanged, but rates of mental retardation were reduced. 